\title{
Practical Compact Routing on Sparse Graphs
}

This paper was downloaded from TechRxiv (https://www.techrxiv.org).

\section{LICENSE}

CC BY 4.0

SUBMISSION DATE / POSTED DATE

$14-11-2021 / 17-11-2021$

CITATION

Hormann, Kai; Gotsman, Craig (2021): Practical Compact Routing on Sparse Graphs. TechRxiv. Preprint. https://doi.org/10.36227/techrxiv.17008186.v1

$\mathrm{DOI}$

10.36227/techrxiv.17008186.v1 


\title{
Practical Compact Routing on Sparse Graphs
}

\author{
Craig Gotsman and Kai Hormann
}

\begin{abstract}
We describe a simple and practical algorithm for compact routing on graphs which admit compact and balanced vertex separators. Using a recursive nested dissection of the $n$ vertex graph based on these separators, we construct routing tables with as few as $O(\log n)$ entries per vertex in a preprocessing step. They support handshaking-based routing on the graph with moderate stretch, where the handshaking can be implemented similarly to a DNS lookup. We describe a basic version of the algorithm that requires modifiable headers and a more advanced version which eliminates this need and provides better stretch. A number of algorithmic parameters control a graceful tradeoff between the size of the routing tables and the stretch. Our routing algorithm is most effective on planar graphs and unit disk graphs of moderate edge/vertex density.
\end{abstract}

Index Terms-Routing, graphs, separators.

\section{INTRODUCTION}

C OMPACT routing arises in communication networks where packets are to be sent between two arbitrary nodes of the network along a short path or route. Call these two nodes $s$ and $t$, the source and target nodes. To facilitate this, each node $v$, or router, of the network contains a routing table that indicates, when a data packet destined for $t$ arrives at $v$, which of the routers connected to $v$ will be the best one to forward the packet to. Obviously, these routing tables should be designed such that they are correct, so always deliver, that is, a packet is always guaranteed to reach its destination $t$ and never get "stuck" at any intermediate router, or, more generally, end up routed in an infinite loop. Additionally, the routing tables should be compact, requiring only $o(n)$ storage per router, where $n$ is the number of nodes, and generate routes with low stretch, where the stretch measures the ratio between the lengths of generated route and the shortest possible route between $s$ and $t$ in the network. There is typically a tradeoff between the routing table storage required by any such scheme (sometimes called the state) and the resulting stretch of the routes. Other relevant, but less important, parameters are the computation time required to build the routing tables (which is typically done in a preprocessing step), the computation time required to determine the next router from the routing table, and the size of the header that each packet must contain with information about the destination node $t$. Historically, compact routing algorithms preferred that the header not be modified during the routing, but more recent algorithms using socalled modifiable or rewritable headers permit this in order to improve the performance of the routing algorithm. Moreover, some schemes achieve smaller routing tables or lower stretch

C. Gotsman is with the New Jersey Institute of Technology, Newark, NJ 07102 USA (e-mail: gotsman@njit.edu).

K. Hormann is with the Università della Svizzera italiana, Lugano, Switzerland (e-mail: kai.hormann@usi.ch).

Manuscript received Xxx nn, 2021; revised Xxx nn, 2021. by admitting a handshaking mechanism at the beginning of each routing procedure.

While it is important to design compact routing schemes for general networks, there are important special cases where the task is easier, and the storage-stretch tradeoff is more manageable. This typically is possible in sparse graphs, those where the number of edges is far less than the maximal $O\left(n^{2}\right)$ possible. In this paper we treat families of graphs which arise in sensor networks, Internet-of-Things and radio and mobile communications scenarios, where nodes are scattered randomly in the plane (or a higher-dimensional Euclidean space) and can communicate with only a subset of the nodes geographically close to them. The underlying mathematical model is the unit disk graph (UDG), where a node is connected to all others within a fixed radius. This models well wifi and bluetooth communication networks. A less realistic model, but simple and mathematically appealing, is the plane graph, where nodes are positioned in the plane and connected by straight-line edges which do not intersect. Plane graphs are also, by definition, sparse.

Sparse graphs such as UDG's and plane graphs have an important property that we will take advantage of: the existence of compact balanced separators [1], [2]. This means that given such a graph $G$ with vertex set $V$ of size $n$, there always exists a subset of the vertices $S$ of size $O(\sqrt{n})$, such that removal of these vertices and the edges incident on them separates the graph into two disjoint components with vertex sets $U_{0}$ and $U_{1}$, both of size at least $n / 3$. The existence of such separators facilitates an efficient "divide-and-conquer" strategy, called nested dissection, which is run on the graph, recursively partitioning it into smaller and smaller pieces. The smallest pieces of the graph, at which the recursion terminates, are called clusters. The resulting separator tree is then used to build compact routing tables which are guaranteed to deliver by generating routes with moderate stretch. In a nutshell, the routing tables guide the routing process such that the packet advances towards a given target vertex $t$ by repeatedly approaching deeper and deeper separators between it and $t$. Once a cluster containing $t$ is reached, the routing proceeds using a conventional routing tree within the cluster.

\section{PREvious WORK}

Compact routing has been investigated since the 1980's, but the first landmark paper, providing a universal scheme for compact routing on general graphs, was by Thorup and Zwick [3] in 2001. They introduce a stretch-3 method with optimal $O(\sqrt{n})$ storage (up to logarithmic factors), establish lower bounds on the storage-stretch tradeoff, and further propose handshake-based variants of their method with presumably optimal tradeoff. These methods remained somewhat 
theoretical until 2010, when the practical implementation of Mao et al. [4] (based on [3]), called S4, appeared. A survey of the progress until 2007 was made by Dom [5].

The special case of planar graphs was first treated by Frederickson and Janardan [6] in 1989. Their algorithm relies on the existence of compact balanced separators for this class of graphs and provides a routing scheme that, for any $0<\epsilon<1 / 3$, uses routing tables of size $O\left(\frac{1}{\epsilon} \log n+n^{1+\epsilon}\right)$ with stretch 7 or $O\left(n^{1 / 3} \log n\right)$ with stretch 3 . Thorup [7] suggests an algorithm trading off storage for stretch: storage $O\left(\frac{1}{\epsilon} \log ^{2} n\right)$ for stretch $1+\epsilon$, and Abraham et al. [8] describe a method requiring $O(\operatorname{polylog}(n))$ storage and guaranteeing some constant stretch (which depends only on the class of graphs considered). The latter also prove a lower bound of $\Omega(\sqrt{n})$ on the storage for stretch 1. Lu's method [9] is applicable for any prescribed set of routing trees per node (including shortest path spanning trees) and requires $O(n)$ storage for stretch 1.

Gupta et al. [10] describe a method based on a compact spanning tree cover of the graph, also derived from compact balanced separators. This method requires a modifiable header which functions as a stack, where data may be pushed on or popped off as the packet proceeds along the route. For planar graphs, this translates into a routing method with $O(\log n)$ spanning trees and stretch 3.

Yan et al. [11] generalized the method of Gupta et al. [10] to UDG's by proving a compact separator theorem for UDG's. This enables an $O(\log n)$ spanning tree cover, with stretch 3. Improvement on separator theorems for UDG's have been obtained over the years, culminating in the results of Carmi et al. [1], who also prove a bound on the size of the separator. Kaplan et al. [12] describe a routing method for UDG's based on the existence of a well-separated pair decomposition of the graph and modifiable headers with a polylogarithmic number of bits. This provides an explicit tradeoff between state and stretch, as follows: for any $\epsilon>0$, there is a routing scheme with stretch $1+\epsilon$ using routing tables of size $O\left(\epsilon^{-5} \log ^{2} D \log ^{2} n\right)$ bits, where $D$ is the Euclidean diameter of the graph. Most recently, Mulzer and Willer [13] construct, for any $\epsilon>0$, a routing scheme that achieves stretch $1+\epsilon$ and storage of $O\left(\log D \log ^{3} n / \log \log n\right)$, where the constant in the O-notation depends on $\epsilon$.

We should mention also the related problem of constructing a distance oracle for a graph. This means building a data structure of size $o\left(n^{2}\right)$ which supports efficient computation of the (approximate) distance between any two vertices of a graph. As in compact routing, here too there is a tradeoff between the size of the data structure, the stretch, and the query runtime. This topic has been an active line of research over the past decades. For planar graphs, Wulff-Nilsen [14] describes an oracle with $O\left(\frac{1}{\epsilon} n(\log \log n)^{2}\right)$ space, $O\left(\frac{1}{\epsilon^{2}}(\log \log n)^{3}\right)$ time and stretch $1+\epsilon$. The most recent result of Gawrychowski et al. [15] presents an oracle of size $O\left(n^{1.5}\right)$ and query time of $O(\log n)$ for stretch 1. More generally, for any $S \in\left[n, n^{2}\right]$, they describe an oracle of size $S$ and query time of $O\left(\max \left(1, n^{1.5} / S\right)\right)$ up to polylogarithmic factors.

Related to compact routing is a body of work on greedy routing, which arises sometimes in the context of robot navigation. This typically means embedding the graph into a higher-dimensional metric space, such that the routing decision (i.e., to which neighbor of the vertex to proceed) is made based only on local information by steepest descent on the (usually Euclidean) distance to the target vertex in this space. As with compact routing, the main challenge in greedy routing is to guarantee delivery, beyond the other questions of the compactness of the representation (i.e., the dimension of the embedding space) and the resulting stretch of the generated paths. A classic result of Bose and Morin [16] states that Euclidean coordinates can support greedy routing on the Delaunay triangulation of any set of points in the plane. Alas, this does not generalize to arbitrary plane graphs. Other notable work on greedy routing on graphs, mostly planar, has appeared over the years [17]-[19].

\section{OUR CONTRIBUTION}

While there has been a lot of theoretical work on the topic of compact routing on general graphs, and also the special cases of planar graphs and UDG's, none of the published papers, except Mao et al. [4], report implementation details, and we are not aware of any independent implementations of these ideas. This could be because they rely on quite sophisticated data structures and algorithms, which are difficult to implement in practice, or because the asymptotic complexity bounds contain large constant factors, which limit the effectiveness of the methods on moderately-sized graphs. Thus, our main contribution is the description of a relatively simple, yet effective, method for compact routing on sparse graphs such as plane graphs and UDG's. The only difficult element of the algorithm is the computation of vertex separators, but this is also relatively easy in practice, since efficient implementations can be found in many publicly available software packages. We also report on the experimental results of our implementation, which can be made available for public use.

\section{THE BASIC IDEA}

In the following subsections we outline the basic version of our routing method, involving a nested dissection of the graph. Its implementation as a set of compact routing tables requires the use of an initial handshaking step to create a header with information about the target $t$ that is attached to the packet. Moreover it requires the header to be modifiable, in order to "remember" some information during the routing process. We later show how to implement a more sophisticated version of the algorithm, which gets by with a non-modifiable header and even improves the stretch relative to the basic method.

\section{A. Nested dissection}

If a connected weighted graph $G=(V, E, w)$ with vertex set $V$ of size $n$, edge set $E \subset V \times V$ of size $m$, and edge weight function $w: E \rightarrow \mathbb{R}^{+}$, is sparse enough to admit compact balanced separators, we may construct a nested (binary) dissection [20] of $G$ represented by a binary tree $T$. Denote by $G(U)$ the subgraph of $G$ defined by the subset of vertices $U \subset V$, inheriting the edges between vertices of $U$ and their weights. The root of $T$ represents $G$ and contains a 


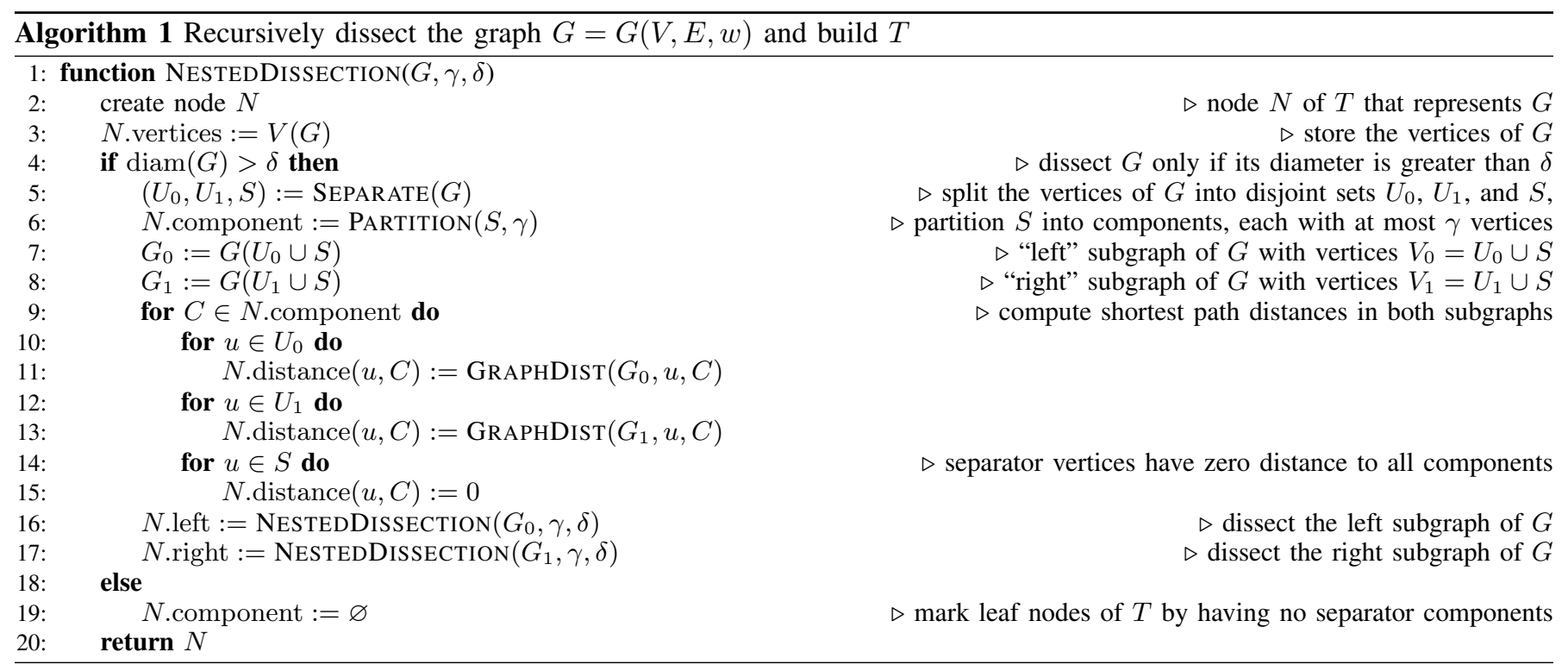

vertex separator $S$ that splits $G$ into two subgraphs, and this is recursively true for all nodes of $T$. More precisely, a node $N$ of $T$ represents a connected subgraph $G(N)=G(V(N))$ of $G$ with vertices $V(N) \subset V$ and a separator $S(N) \subset V(N)$, such that $V(N) \backslash S(N)$ consists of exactly two connected components, with vertex sets $U_{0}(N)$ and $U_{1}(N)$, respectively. Let $V_{0}(N)=U_{0}(N) \cup S(N)$ and $V_{1}(N)=U_{1}(N) \cup S(N)$, and denote by $G_{0}(N)$ and $G_{1}(N)$ the subgraphs of $G$ with vertex sets $V_{0}(N)$ and $V_{1}(N)$, respectively. To facilitate routing, in this node $N$ we store the list of separator vertices $S(N)$ and a set of (weighted) graph distances between $S(N)$ and the vertices $V_{0}(N)$ within $G_{0}(N)$ and between $S(N)$ and the vertices $V_{1}(N)$ within $G_{1}(N)$, respectively. In some cases it is beneficial to further partition $S(N)$ into a number of disjoint components $C \subseteq S(N)$ with at most $\gamma$ vertices each, and consider instead the vector of distances between the vertices and these components. The two children of $N$ then represent $G_{0}(N)$ and $G_{1}(N)$ respectively. We say that $s, t \in V(N)$ are separated by $S(N)$, if $s \in V_{0}(N)$ and $t \in V_{1}(N)$, or viceversa. We further say that $S(N)$ is a strong separator of $s$ and $t$, if $s \in U_{0}(N)$ and $t \in U_{1}(N)$, or vice-versa (namely, both $s$ and $t$ are not on $S(N)$.)

Ultimately, the recursion terminates when the subgraph of $G$ represented by a node is small enough in some meaningful sense. The simplest way to do this is to terminate once the level of the recursion exceeds some specified depth, as the size (i.e., number of vertices) of a subgraph at depth $k$ will typically scale like $2^{-k} n$. More sophisticated termination criteria could take into account the actual size of the subgraph, or its diameter. We chose to use the diameter parameter, so the leaves of $T$ represent small subgraphs $G^{\prime}$ of $G$ with bounded diameter $\operatorname{diam}\left(G^{\prime}\right) \leq \delta$ that we call clusters. Note that as the dissection generates subgraphs having the vertices of the separator in common, each vertex $v$ of $G$ may belong to more than one cluster. We informally call the separators at nodes close to the root of the tree $T$ high-level separators and the ones close to the leaves deep separators. If the separators are balanced, then the depth of $T$ is $O(\log n)$.
We say that a node $N$ of $T$ and its separator $S(N)$ are associated with all vertices $v \in V(N)$. Vice-versa, each vertex $v \in V$ is contained in a subset of the leaves of $T$, so we may associate with $v$ the separator subtree $T_{v}$ rooted at the root of $T$ whose leaves contain $v$, but without the leaves themselves, as well as all the nodes of $T_{v}$ and the separators represented by these nodes.

\section{B. Vertex separators}

Nested dissection depends on the existence of compact balanced separators and an efficient algorithm to compute such separators. The celebrated theorem of Lipton and Tarjan [2] guarantees that every $n$-vertex planar graph contains a separator of size $O(\sqrt{n})$, such that the size of each separated component is between $n / 3$ and $2 n / 3$. Similar theorems exist also for UDG's [1]. A simple and common way that we use to generate good separators, which usually give components with this property, is the spectral method [21], where the socalled Fiedler eigenvector of the graph Laplacian matrix is used. This vector is negative on approximately half the vertices and the edges connecting the negative vertices with positive ones are a compact edge cut of $G$. This implies that the positive (or negative) endpoints of these edges are a compact vertex separator of the graph. More elaborate methods exist (see survey by Buluc et al. [22]), some implemented quite efficiently in the MeTiS software package [23].

\section{The separator tree}

Algorithm 1 describes the recursive function NEstedDisSECTION that constructs the separator tree $T$ for a graph $G(V, E, w)$. Each interior node $N$ of $T$ contains the components of a separator, the distances from the subgraph vertices to each separator component, and pointers to the nodes representing the two subgraphs induced by the separator. The leaf nodes of $T$ store just the vertices of the corresponding cluster. $\operatorname{Separate}(G)$ is the graph separation routine on $G$ that returns two separate components $U_{0}, U_{1} \subset V(G)$ and 

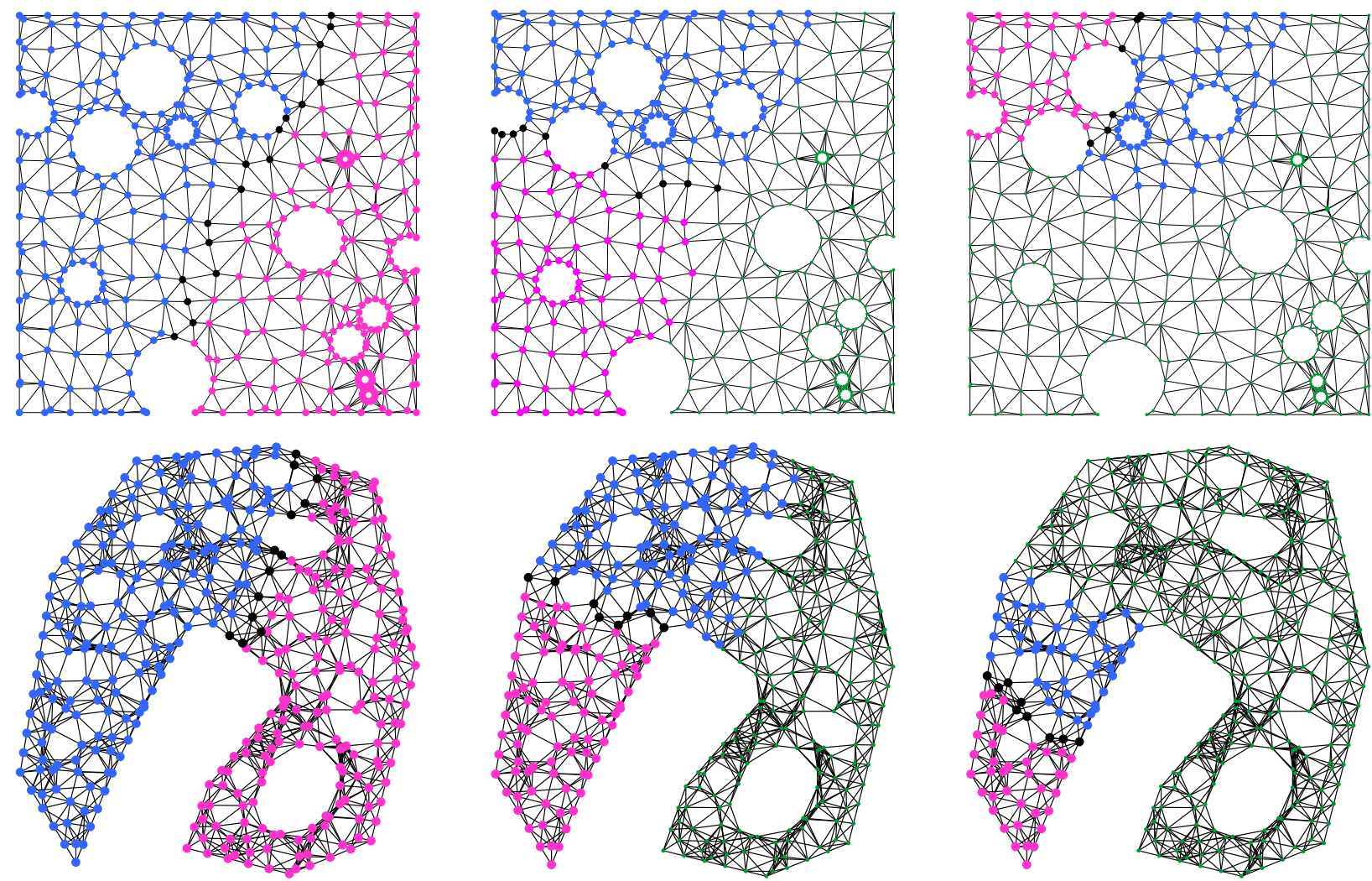

Fig. 1. The nested dissection process: three levels of dissection for the planar graph PLANAR (top) and the unit disk graph UDG_04_07 (bottom). Magenta and blue mark the vertices in each of the two components, and black vertices are the separator vertices at each level.

a vertex separator $S \subset V(G)$. PARTition $(S, \gamma)$ partitions a set of vertices $S$ into components (i.e., subsets close to each other) no larger than $\gamma$. GRAPHDist $(G, u, W)$ is a routine that computes the vector of weighted shortest path distances in $G$ between the vertex $u \in V(G)$ and the vertex subset $W \subset V(G)$. Figures 1 and 2 show some examples of this nested dissection process.

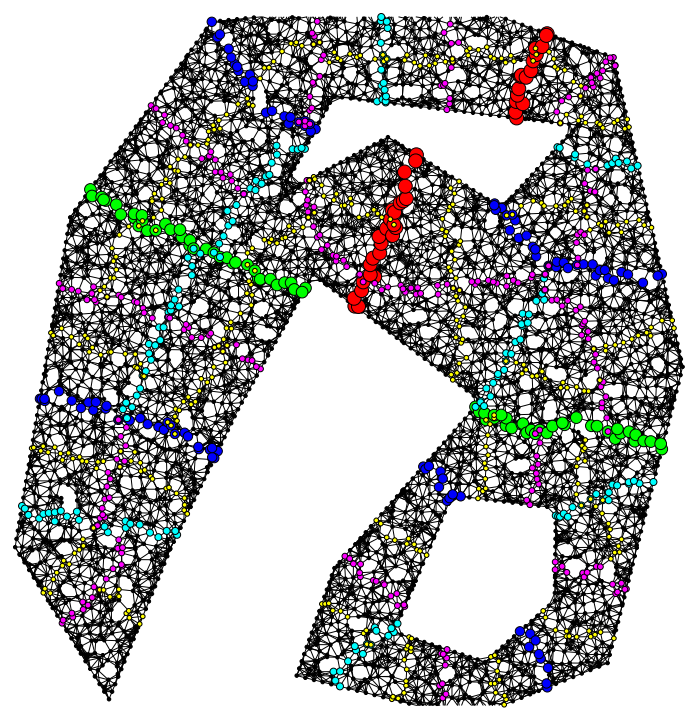

Fig. 2. Complete set of vertex separators on the unit disk graph UDG_01_0175 at 6 levels, color-coded (red, green, blue, cyan, magenta, yellow) and size decreasing with depth.
Figure 3 illustrates the following observations that characterize the separator tree $T$ and the separator subtrees $T_{v}$ :

- Each vertex $v \in V$ is contained in at least one leaf of $T$ (a cluster), but possibly in more than one.

- $T_{v}$ consists of all paths in $T$ from the root to the leaves containing $v$ (without the leaves themselves). In most cases, these paths coincide close to the root, so that the upper part of $T_{v}$ is a single path, which bifurcates at a node with a separator containing $v$. Hence, if $v$ is contained in a high-level separator, then the path bifurcates close to the root.

- Given two distinct vertices $v, w \in V$, the separator subtree $T_{v} \cap T_{w}$ is the union of the paths from the root to the least common ancestor (LCA) of any two leaves in $T$, one containing $v$ and one containing $w$.

- The LCA in $T$ of any two leaves containing two distinct non-adjacent vertices $v, w \in V$ contains a separator between the two. Any such separator which has no other separator among its children is a strong separator (otherwise it is not). Each subtree of a node containing a strong separator contains only $v$ or $w$ exclusively. We will take advantage of this property in Lemma 1 below.

\section{The basic routing scheme}

Once the graph $G$ has been recursively decomposed into a tree $T$, it is easy to see how routing from $s$ to a given target vertex $t$ may be achieved. By definition, if $s$ and $t$ are separated by vertex separator $S$, then any path between $s$ and $t$ must pass 

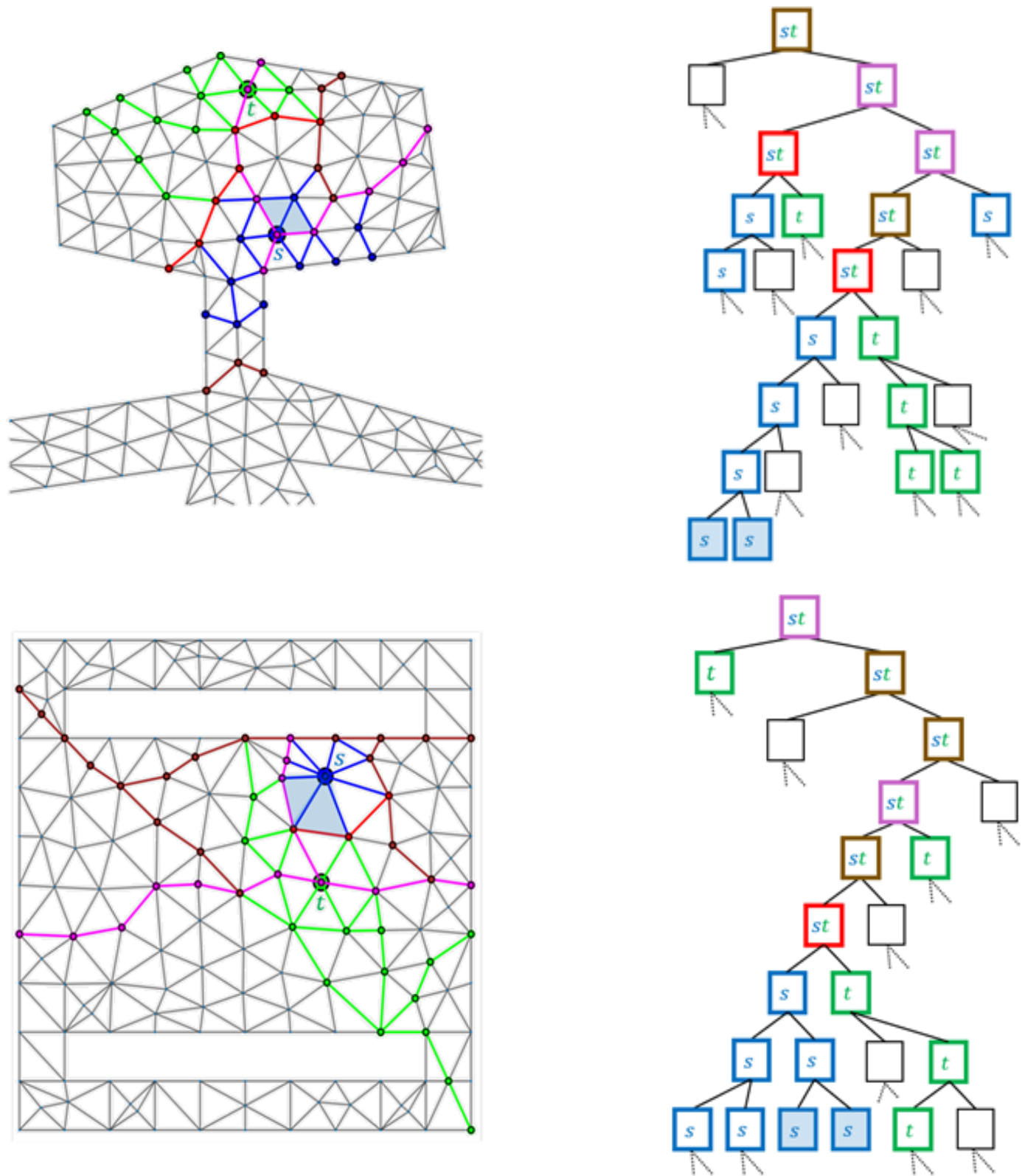

Fig. 3. Two vertices, $s$ (blue) and $t$ (green), in two plane graphs (left) and their separators in the separator tree $T$ (right). The root of $T$ is at depth 0 . Note that all the separators happen to be edge paths in the graph and the dissection is done down to the level of (diameter 1) cliques which are single edges or triangles. Each interior tree node represents a separator and is labeled with $s$ and $t$ depending on whether either of the two vertices is associated with this separator. Each node is colored by the type of separator. Blue/green are those separators associated with only the blue/green vertex, respectively. Brown are those associated with both vertices but do not separate between them (at depths 0,3 on the top and at depths 1,2,4 on the bottom). Magenta are those associated with both vertices and separate them (at depths 1,2 on the top and at depths 0,3 on the bottom), and red are strong separators of $s$ and $t$ (at depth 2,4 on the top and at depth 5 on the bottom). To illustrate, two of the leaves corresponding to the two blue shaded triangles containing the blue vertex are also shaded in blue.

through $S$ at some point. Thus a promising routing procedure, as illustrated in Figure 4, is to repeatedly move from $s$ towards $S$. Once $S$ is crossed at vertex $\sigma$, this idea repeats with a new separator deeper than $S$ in $T$, separating between $\sigma$ and $t$. This again repeats until the current vertex $s$ is not separated at all from $t$, implying that it is contained in some cluster of the nested dissection as $t$ is. From this point on, routing proceeds by standard routing tree methods within the small cluster. This routing algorithm is described in Algorithm 2. If $S$ consists of just one component, then it is not hard to show that this algorithm always routes along the shortest path from $s$ to $\sigma$, which is the closest point on the deepest separator $S$ between $s$ and $t$, and that this process repeats with $s$ replaced by $\sigma$ until a cluster containing $t$ is reached. More precisely, $S$ is always the "leftmost" deepest separator, if there happens to be more than one such separator. In the case that $S$ is partitioned into multiple components, the algorithm routes to the component $C$ of $S$ with the smallest sum of distances to $s$ and $t$.

\section{COMPact Routing}

While the basic routing method described in Algorithm 2 is quite straightforward, the devil is in the details. The main 


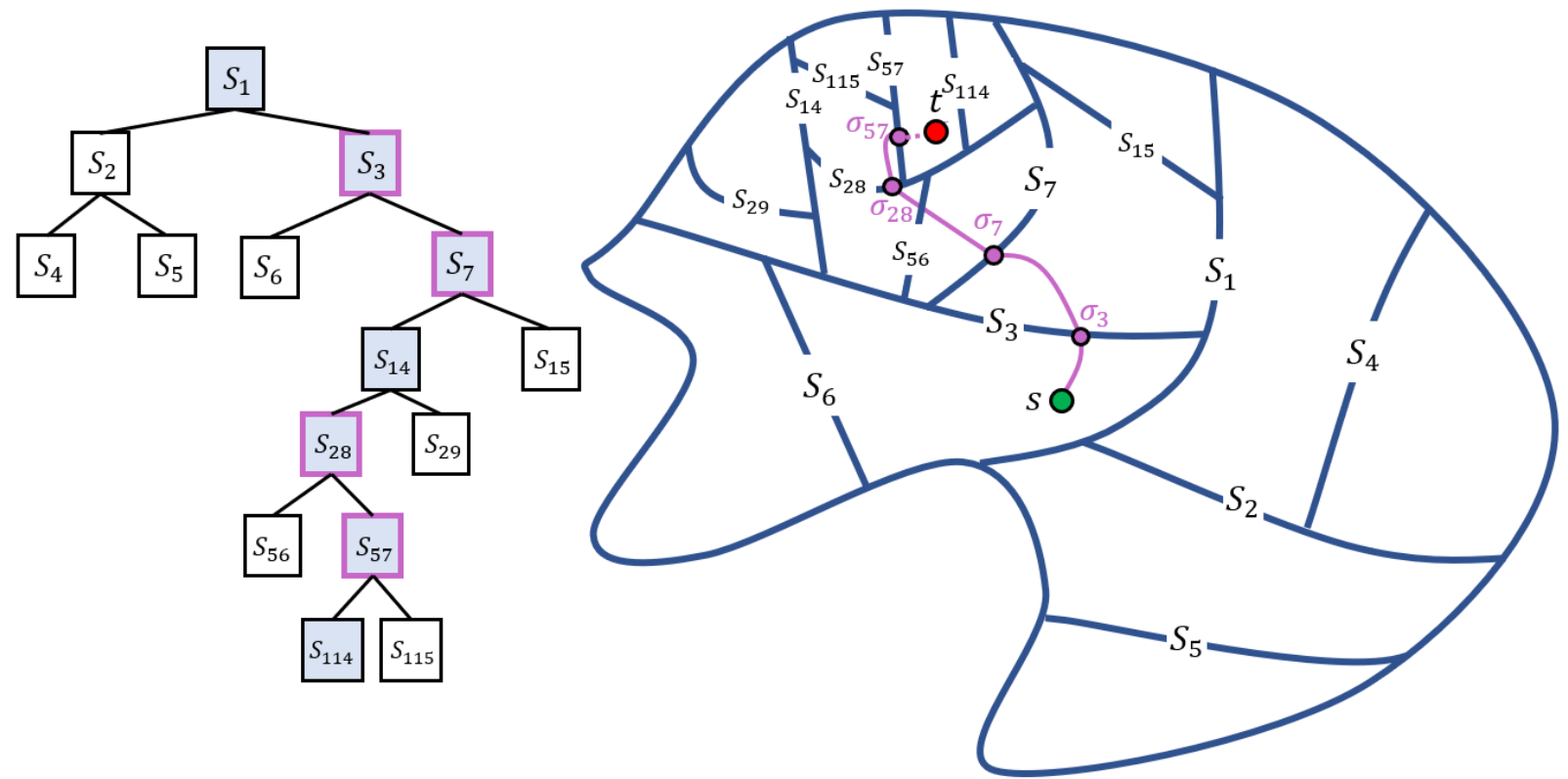

Fig. 4. The basic routing scheme: The binary tree $T$ (left) is part of the full binary tree representing the nested dissection of the graph (right). The separators $S_{i}$ (not partitioned into components in this example) are labeled in the standard binary tree convention, where $2 i$ and $2 i+1$ are the labels of the descendants of node $i$. Blue nodes from root to leaf in $T$ are those associated with target vertex $t$. The node containing $S_{3}$ is the LCA of the nodes associated with $s$ and $t$, thus the first separator used in the routing process. The vertices $\sigma_{i}$ are the crossing points of relevant $S_{i}$ when routing to $t$. Purple bordered nodes in $T$ are those which strongly separate $s$ and $\sigma_{i}$ from $t$, thus are used to route from $s$ to $t$.

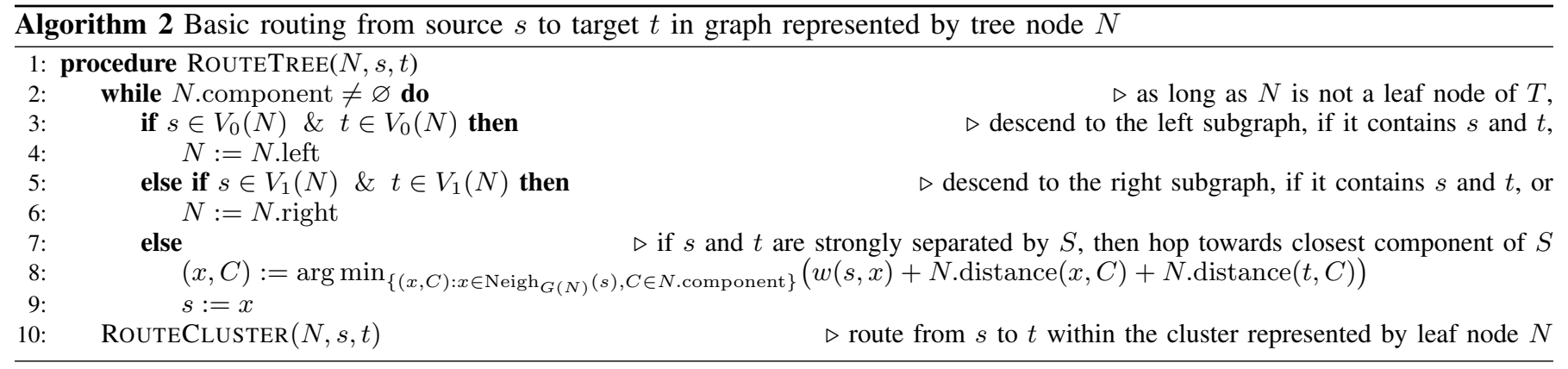

challenge is to implement this method through independent (compact) routing tables at each graph vertex, and provide algorithm parameters, which control the storage-stretch tradeoff. This is non-trivial, as care must be taken that the routing does not get confused when a separator is approached, especially if it is "thick" and must be crossed without the route ending up in a loop (e.g., a loop of length one, i.e., a "sink"). Algorithm 2 achieves this by making routing decisions based on a "memory" of the node $N$ in $T$ that contains the current target separator $S(N)$. While this information could be stored and updated in a modifiable header during the routing, we would like to avoid that.

In this section we show how to circumvent this requirement by being a little more careful in choosing the separator to route to. This makes the algorithm just a little more complicated but has the additional benefit of achieving better stretch than the basic algorithm. Moreover, we note that the routing decision in each node requires access to the distances between $t$ and the components of the separators associated with $t$ (cf. line 8 of Algorithm 2). We describe how to retrieve this information in an initial handshaking step and store it in the packet header.

\section{A. Distance estimates}

Given a graph $G=(V, E, w)$, the length of a path of edges is defined as the sum of the weights of those edges, and the distance $d_{G}(s, t)$ between two vertices $s, t \in V$ is defined as the length of the shortest path between $s$ and $t$ within $G$. This notion of distance between vertices may be extended to the distance between two sets of vertices $\mathcal{S}$ and $\mathcal{T}$ as

$$
d_{G}(\mathcal{S}, \mathcal{T})=\min _{s \in \mathcal{S}, t \in \mathcal{T}} d_{G}(s, t) .
$$

Note that if $G^{\prime}$ is a subgraph of $G$, then

$$
d_{G}(s, t) \leq d_{G^{\prime}}(s, t)
$$

for all $s, t \in V\left(G^{\prime}\right)$, because it may happen that the shortest path between $s$ and $t$ in $G$ is not entirely in $G^{\prime}$. We further denote the neighbours of $s$ within $G^{\prime}$ by

$$
\operatorname{Neigh}_{G^{\prime}}(s)=\left\{u \in V\left(G^{\prime}\right):(s, u) \in E\right\} .
$$

Recall that the key quantity in our routing method, stored in the separator tree, is the distance between a vertex $v$ and a separator component $C$. Note that for two vertices 


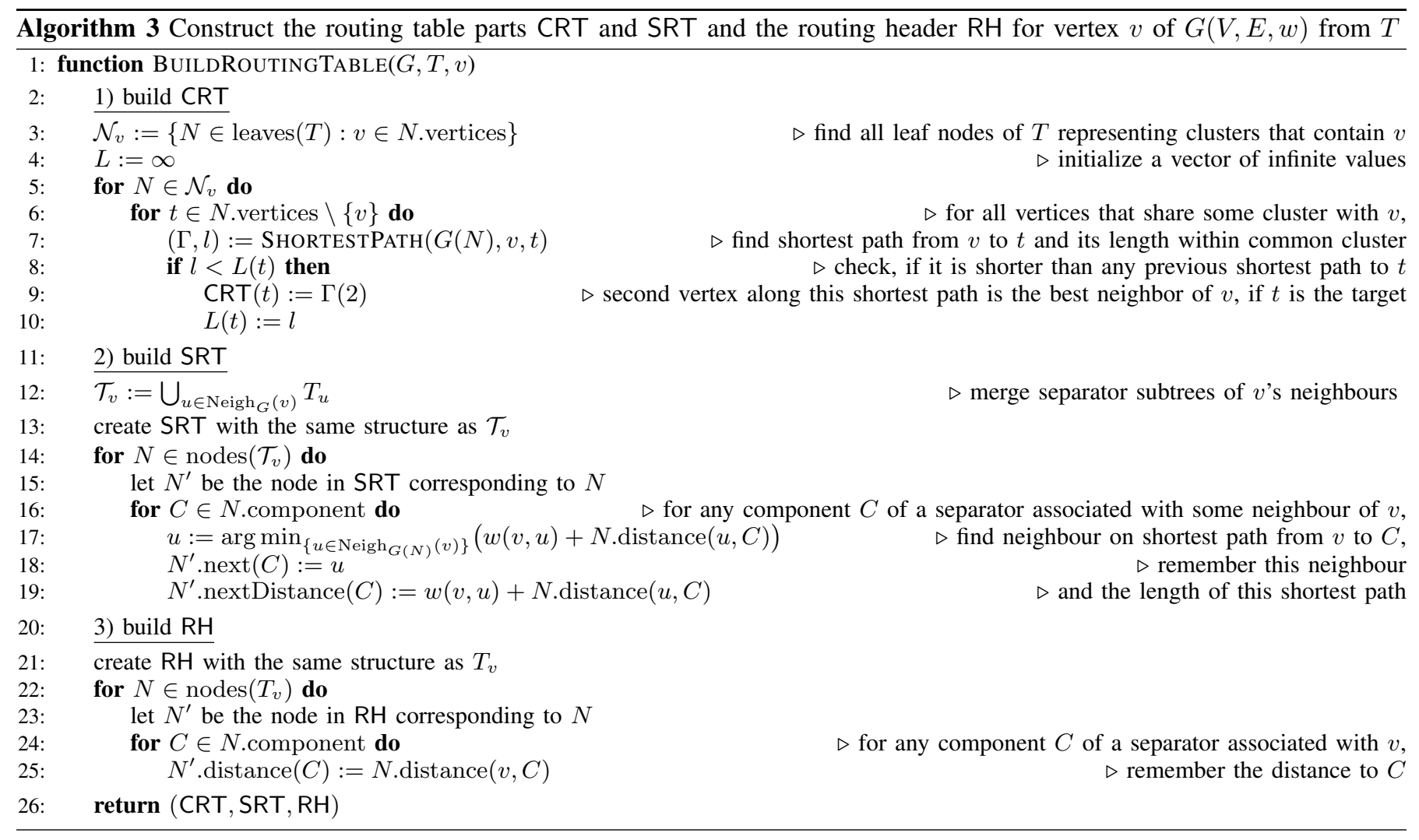

$s, t \in V(G)$, if they are separated by $S$, partitioned into certain $C$, in two connected components $G_{0}$ and $G_{1}$, then a reasonable approximation of the distance $d_{G}(s, t)$ is the estimated distance through $S$,

$$
e_{G}^{S}(s, t)=\min _{C \subseteq S}\left\{d_{G_{0}}(s, C)+d_{G_{1}}(t, C)\right\},
$$

where we assume without loss of generality that $s \in V_{0}(S)$ and $t \in V_{1}(S)$. More precisely, it follows from the triangle inequality that the estimated distance is a lower bound on the true distance,

$$
e_{G}^{S}(s, t) \leq d_{G}(s, t) .
$$

As we shall see later, these distance estimates, in particular from the target $t$, will guide the routing towards $t$.

Since the separators generated by the nested dissection are in one-to-one correspondence with the internal nodes of the tree $T$, specifying a separator $S$ uniquely determines the node $N(S)$ that contains it, thus also the subgraph $G(N)$ that it separates into $G_{0}(N)$ and $G_{1}(N)$. As such, we may omit the subscript $G$ and use the notation $e_{S}$ instead of $e_{G}^{S}$.

\section{B. The routing tables}

The first step in the routing method is the offline construction of a routing table for each graph vertex $v$. Each routing table should contain enough information to determine which of $v$ 's neighbors to proceed to given a target vertex $t$ and should be such that a packet is always guaranteed to eventually arrive at $t$ without getting stuck in an infinite loop of any sort. The routing table of $v$ consists of two parts, the cluster routing table $\mathrm{CRT}(v)$ and (a description of) the separator routing tree $\operatorname{SRT}(v)$, generated by Algorithm 3 .
CRT is a standard list of pairs $\left(t_{i}, u_{i}\right)$ which indicates that if the target vertex is $t_{i}$, then the routing should proceed to neighbor $u_{i}$ of $v$. The vertex $u_{i}$ is just the predecessor of $v$ in the shortest path tree rooted at $t_{i}$ within the cluster that contains $v$ and $t_{i}$. In principle it is possible to build such a table for all possible targets in the graph, but then the size of the table would be a prohibitive $O(n)$. So in practice, this table contains routing information in CRT only for the small number of vertices which happen to be in a cluster which also contains $v$. Moreover, we pay attention to store the neighbour on the shortest of all shortest paths, in case $v$ and $t_{i}$ are in more than one common cluster. By definition of the clusters, the hop distance of these vertices from $t$ is at most $\delta$.

For all other vertices $t$ more distant from $v$, there is no explicit information in the cluster routing table on how to get closer to $t$. This is where the more sophisticated part of the routing table, the SRT, comes in. In this case, routing is in the direction of a separator $S$ between $v$ and $t$, building on the separation property, namely that any path between $v$ and $t$ must intersect $S$, or rather one of its components at some point. Thus we must ensure that all other target vertices have at least one separator between them and $v$ and information about the components $C$ of this separator is present in $v$ 's SRT. More precisely, SRT contains for each separator component $C$ associated with some neighbor of $v$, the length of the shortest path from $v$ to $C$ passing through a neighbour $u$ of $v$ associated with $C$, as well as $u$ itself. To store this information, $\operatorname{SRT}(v)$ needs to have the same structure as the union $\mathcal{T}_{v}=\bigcup_{u \in \operatorname{Neigh}_{G}(v)} T_{u}$ of the separator subtrees of $v$ 's neighbours. 


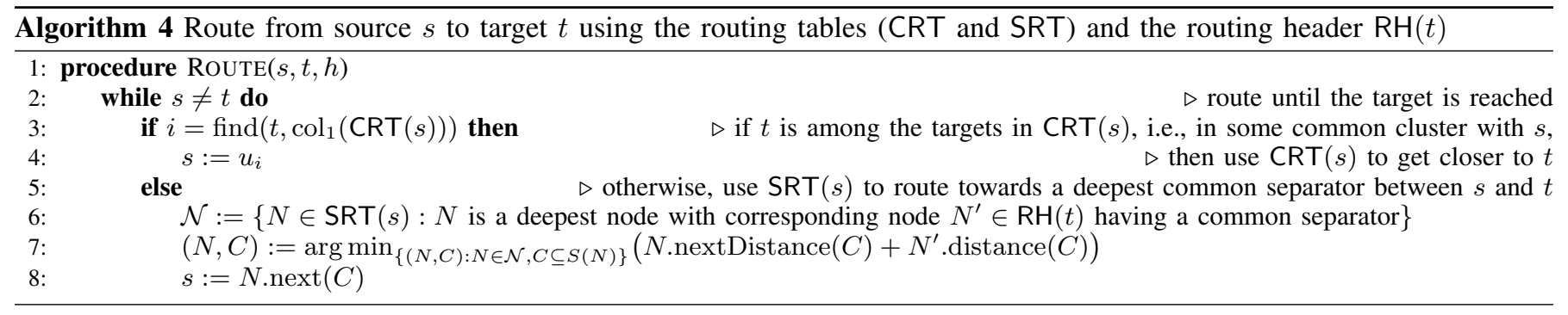

\section{The routing header}

Given a target vertex $t$, our routing algorithm relies on a header that characterizes $t$ and provides all the information required throughout the routing procedure to guide the routing towards $t$. In essence, these are the distances of $t$ to all the components of its associated separators, which are contained in the separator subtree $T_{t}$ and copied into the much smaller structure $\mathrm{RH}(t)$ (see Algorithm 3). At the beginning of the routing procedure, this routing header $\mathrm{RH}(t)$ is appended to the packet. To this end, we could store each routing header in the corresponding network node and implement a handshaking mechanism by which $s$ retrieves $\mathrm{RH}(t)$ from $t$. Alternatively, we could store all routing headers in a small number of "hub" nodes and in each node the routing header of the nearest hub, so that our routing algorithm itself can be used for retrieving $\mathrm{RH}(t)$, similar to a DNS lookup in TCP/IP. Other variants are possible and may depend on the particular application, but in any case the resulting overhead is expected to be small in comparison to the cost of routing a long stream of packets. Hence, we excluded the handshake in our implementation and the results reported below.

\section{The routing algorithm}

The routing of a packet from $s$ to $t$ proceeds by using the routing table at $s$ to determine which of the neighbors of $s$ to forward the header and packet to, as described in Algorithm 4. As we will see later, Algorithm 4 is more sophisticated than the basic Algorithm 2. The former, by definition, always proceeds down through the tree, whereas the latter also allows lateral moves in the tree, if that brings the packet closer to the target. After all, this is not so surprising, because without the "memory" of the current separator, the algorithm cannot simply "descend" in the underlying separator tree, but rather has to consider all possible paths in the tree that lead to a cluster containing $t$.

Before we start, let us establish a simple, but key property of the separator tree (cf. Figure 3).

Lemma 1. Let $u, v \in V$ be two distinct vertices, $N$ a deepest node of $T_{u} \cap T_{v}$, and $S$ the separator at $N$. Then, $u$ and $v$ are either strongly separated by $S$ or in a common cluster.

Proof. If $u$ and $v$ are not strongly separated by $S$, then we can assume without loss of generality that $u, v \in V_{0}(N)$. But as $N$ is a deepest node among all nodes containing both $u$ and $v$, this is possible only if $G_{0}(N)$ is a cluster.

For a fixed target vertex $t$ and any vertex $u \in V$ that is not in a common cluster with $t$, let $k_{u}$ be the depth of the deepest nodes in $T_{u} \cap T_{t}$ and $\mathcal{S}_{u}$ be the set of deepest separators between $u$ and $t$ corresponding to these deepest nodes. Note that deepest separators are necessarily strong separators by Lemma 1. We denote the smallest distance estimate (1) based on these deepest separators by $e_{u}=\min _{S \in \mathcal{S}_{u}} e_{S}(u, t)$, and let $S_{u}=\arg \min _{S \in \mathcal{S}_{u}} e_{S}(u, t)$ be a corresponding separator. Further let $N_{u}$ be the node associated with $S_{u}$ and $C_{u}$ be a component of $S_{u}$ with $e_{u}=d_{G_{0}}\left(u, C_{u}\right)+d_{G_{1}}\left(t, C_{u}\right)$, where $G_{0}=G_{0}\left(N_{u}\right)$ and $G_{1}=G_{1}\left(N_{u}\right)$, assuming without loss of generality that $u \in V\left(G_{0}\right)$ and $t \in V\left(G_{1}\right)$. In other words, among all estimated distances between $s$ and $t$ through one of the components of the separators in $\mathcal{S}_{u}$, the one through $C_{u}$ is the smallest.

With these definitions, we are now ready to prove that the separator routing trees $\operatorname{SRT}(v)$ can be used to repeatedly bring the packet closer to a cluster that contains $t$, either by descending a level in $T$ or by reducing the remaining estimated distance to $t$. Once such a cluster is reached, the final delivery to $t$ is handled by the cluster routing tables $\operatorname{CRT}(v)$.

Theorem 1. For any vertex $s$, let $v$ be the neighbour of $s$ to which the ROUTE procedure moves in line 8 of Algorithm 4. Then, either $k_{v}>k_{s}$ or $k_{v}=k_{s}$ and $e_{v} \leq e_{s}-w(s, v)$.

Proof. Let $S_{s}$ be the deepest separator in $T_{s} \cap T_{t}$ as defined above and $N_{s}$ be the node associated with $S_{s}$. Since we only get to line 8 in Algorithm 4 as long as $s$ is not in a common cluster with $t$, we conclude from Lemma 1 that $S_{s}$ is a strong separator of $s$ and $t$, and can hence assume without loss of generality that $s \in U_{0}\left(N_{s}\right)$ and $t \in U_{1}\left(N_{s}\right)$. Let $r$ be the closest vertex to $s$ in $C_{s}$, that is, $d_{G_{0}}(s, r)=d_{G_{0}}\left(s, C_{s}\right)$, where $G_{0}=G_{0}\left(N_{s}\right)$ and $G_{1}=G_{1}\left(N_{s}\right)$. Further let $\Gamma$ be a shortest path from $s$ to $r$ within $G_{0}$, and $u \in \operatorname{Neigh}_{G_{0}}(s)$ be the neighbor of $s$ along $\Gamma$ (see Figure 5).

We first observe that

$$
e_{s}=d_{G_{0}}\left(s, C_{s}\right)+d_{G_{1}}\left(t, C_{s}\right)=d_{G_{0}}(s, r)+d_{G_{1}}\left(t, C_{s}\right),
$$

by the definition of $r$, and

$$
d_{G_{0}}\left(u, C_{s}\right)=d_{G_{0}}(u, r)=d_{G_{0}}(s, r)-w(s, u),
$$

by the definition of $u$ and the fact that the path from $u$ to $r$ along $\Gamma$ is a shortest path, too. Moreover,

$$
k_{u} \leq k_{v},
$$

because $k_{v}$ is the depth of deepest node in the tree $\mathcal{T}_{s} \cap T_{t}$, which contains $T_{u} \cap T_{t}$ by definition.

Since $s \in U_{0}\left(N_{s}\right)$ implies $u \in V_{0}\left(N_{s}\right)=U_{0}\left(N_{s}\right) \cup S_{s}$, we know that $S_{s}$ is also a (possibly weak) separator of $u$ and $t$ corresponding to some node in $T_{u} \cap T_{t}$, which is not necessarily 


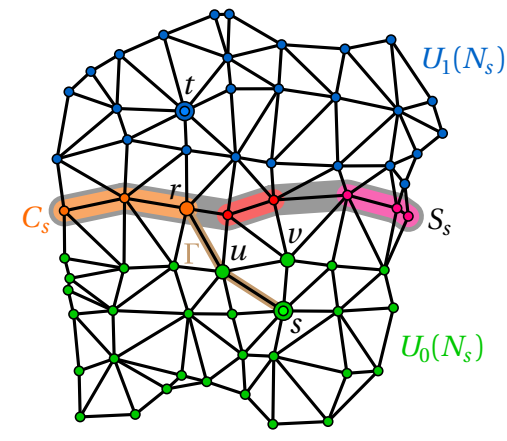

Fig. 5. Notation used in the proof of Theorem 1.

among the deepest nodes, hence $k_{s} \leq k_{u}$. If $k_{s}<k_{u}$, then (4) implies that the theorem holds, and likewise in the case that $k_{u}<k_{v}$. It remains to consider the case $k_{s}=k_{u}=k_{v}$. In this case, $S_{s}$ is among the deepest separators in $\mathcal{S}_{u}$ and

$$
\begin{aligned}
e_{u} & =\min _{S \in \mathcal{S}_{u}} e_{S}(u, t) \leq e_{S_{s}}(u, t) \\
& \leq d_{G_{0}}\left(u, C_{s}\right)+d_{G_{1}}\left(t, C_{s}\right)=e_{s}-w(s, u),
\end{aligned}
$$

where the last equality follows from (2) and (3). Our goal now is to show that the estimated distance $w(s, u)+e_{u}$ from $s$ to $t$ via $u$ cannot be smaller than the estimated distance $w(s, v)+e_{v}$ via $v$, since that would contradict the choice of $v$ as the best neighbor to route to. To this end, recall that $e_{v}$ is the smallest estimated distance from $v$ to $t$ through any deepest separator in $\mathcal{S}_{v}$, which are among the deepest separators in $\mathcal{T}_{s} \cap T_{t}$. Therefore, by the definition of N.nextDistance in line 19 of Algorithm 3,

$$
\begin{aligned}
w(s, v)+e_{v} & \leq w(s, v)+d_{G_{0}(N)}(v, C)+d_{G_{1}(N)}(t, C) \\
& =N \cdot \operatorname{nextDistance}(C)+d_{G_{1}(N)}(t, C),
\end{aligned}
$$

where $N$ and $C$ are the optimal deepest node and component selected in line 7 of Algorithm 4. Since also the separators in $\mathcal{S}(u)$ are among the deepest separators in $\mathcal{T}_{s} \cap T_{t}$, it follows from the definition of $N, C, N$.nextDistance, and $e_{u}$ that

$$
\begin{aligned}
& N . \text { nextDistance }(C)+d_{G_{1}(N)}(t, C) \\
& \quad \leq N_{u} \cdot \operatorname{nextDistance}\left(C_{u}\right)+d_{G_{1}\left(N_{u}\right)}\left(t, C_{u}\right) \\
& \leq w(s, u)+d_{G_{0}\left(N_{u}\right)}\left(u, C_{u}\right)+d_{G_{1}\left(N_{u}\right)}\left(t, C_{u}\right) \\
& \quad=w(s, u)+e_{u} .
\end{aligned}
$$

Overall, this gives the desired inequality

$$
w(s, v)+e_{v} \leq w(s, u)+e_{u} \leq e_{s},
$$

with the second equality following from (5).

Note that the most important step in Algorithm 4, which is also key for the proof of Theorem 1, is selecting the deepest separator. To illustrate this, consider the following simple scenario where not choosing the deepest separator results in the router entering a loop:

Suppose that $r$ is the closest neighbor of $s$, that is, $w(s, r) \leq w(s, u)$ for all $u \in \operatorname{Neigh}_{G}(s)$, and $s$ is also the closest neighbour of $r$. Further assume that $s, r$, and $t$ all lie on some separator $S$ represented by node $N$, but
TABLE I

GRAPHS USED IN OUR EXPERIMENTS

\begin{tabular}{|c|c|c|}
\hline Name & \#Vertices $(n)$ & \#Edges $(m)$ \\
\hline PLANAR & 416 & 1,041 \\
UDG-SMALL1 & 379 & 876 \\
UDG-SMALL2 & 379 & 1,222 \\
UDG-SMALL3 & 379 & 1,650 \\
UDG-SMALL4 & 379 & 2,181 \\
UDG-SMALL5 & 379 & 2,809 \\
UDG-MEDIUM & 4,563 & 19,694 \\
UDG-LARGE & 26,542 & 113,924 \\
\hline
\end{tabular}

$s$ and $t$ are not in a common cluster. For any component $C \subseteq S$, it follows from Algorithms 1 and 3 that $N$.next $(C)=r$ and $N$.nextDistance $(C)=w(s, r)$ in $\operatorname{SRT}(s)$ and $N^{\prime}$.distance $(C)=0$ in $\operatorname{RH}(t)$, so that Route will choose $r$ as the next vertex to hop to. Alas, once we are at $r$, symmetry implies that we will route back to $s$, and a loop is formed. The problem in this scenario is that $S$ is not a strong separator of $s$ and $t$. However, if ROUTE selects the deepest separator, which strongly separates $s$ and $t$ by Lemma 1 , then the symmetry is broken and the routing procedure eventually reaches a cluster that contains $t$, as stated by Theorem 1 .

We now prove the central property of our routing scheme, namely, that Algorithm 4 delivers.

\section{Corollary 1. The procedure ROUTE terminates at $t$.}

Proof. We first observe that the else-branch in the while loop is executed only a finite number of times. This follows directly from Theorem 1, by noticing that the pair $\left(k_{s}, e_{s}\right)$ decreases strictly monotonically with respect to lexicographic order in each step of the while loop. Hence, no vertex can be visited twice, and since the number of vertices is finite, this part of the procedure terminates when $s$ enters a cluster containing $t$.

From this point on, the definition of the cluster routing tables (cf. Algorithm 3) guarantees that we route along a path towards $t$ that is at most as long as the shortest of all shortest paths from $s$ to $t$ within the clusters that contain $s$ and $t$. Indeed, if $N$ is the leaf node of $T$ that represents the cluster $V(N)$ containing this shortest path $\Gamma$ from $s$ to $t$ and $u$ is the neighbor of $s$ to which the ROUTE procedure moves in line 4 of Algorithm 4, then $u \in V(N)$ and the shortest path from $u$ to $t$ in the cluster $V(N)$ is the one following $\Gamma$. Hence, $\mathrm{CRT}(u)$ will continue to route along $\Gamma$, unless there is an even shorter path from $u$ to $t$ in some other cluster.

\section{EXPERIMENTAL RESULTS}

We have implemented all components of our routing method in MATLAB and run it on a number of sample inputs, consisting of planar graphs and UDG's of varying size and edge density. Table I provides the specifications of these graphs and Figure 6 shows some of them. The first set of UDG's (UDGSMALL) were generated by scattering $n=379$ points randomly within a non-convex polygonal region with two "holes", bounded by the unit square, and then connecting with an edge any two vertices whose distance was less than a parameter, which took values in $\{0.05,0.06,0.07,0.08,0.09\}$, resulting in graphs with identical vertex sets, but with $m=876,1,222$, 


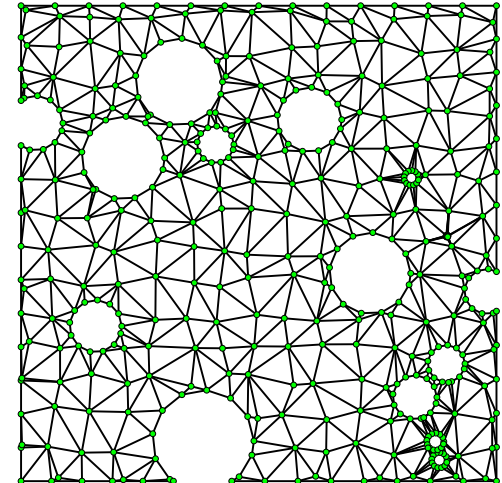

PLANAR

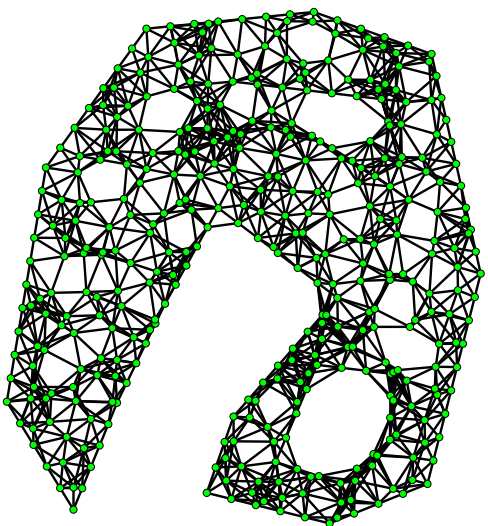

UDG-SMALL3

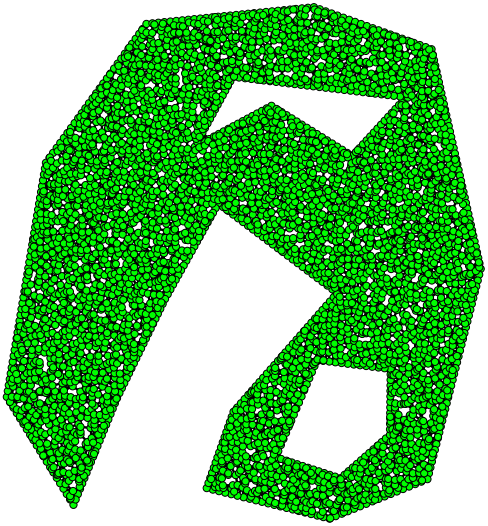

UDG-MEDIUM

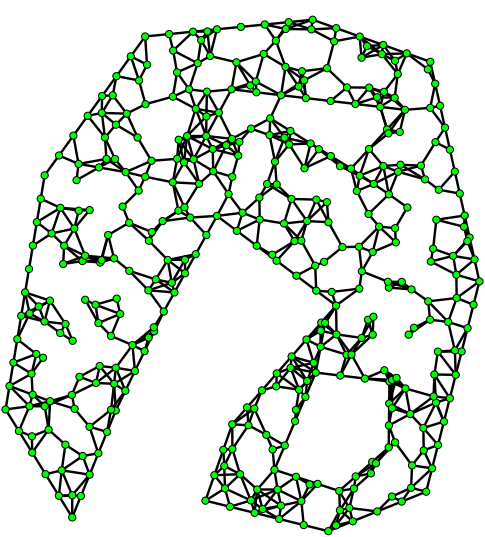

UDG-SMALL1

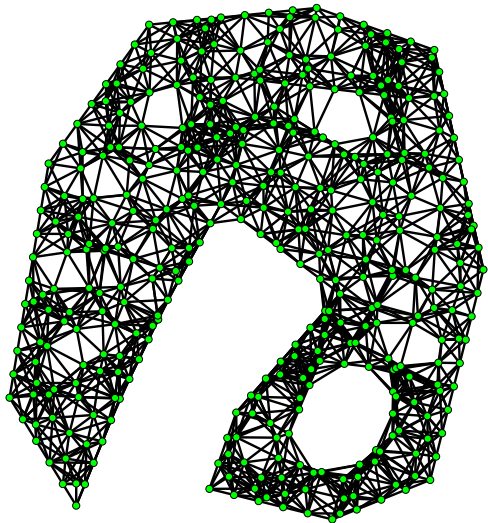

UDG-SMALL4

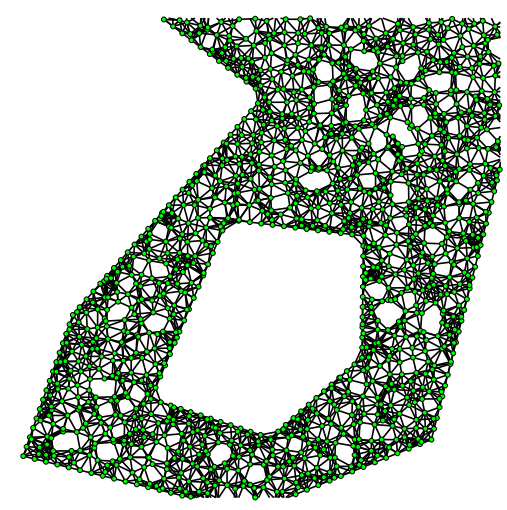

closeup of UDG-MEDIUM

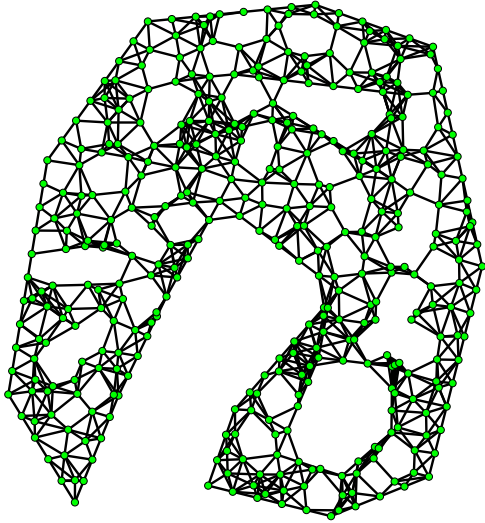

UDG-SMALL2

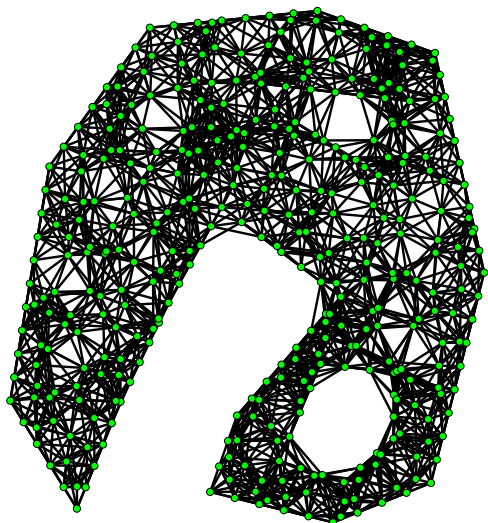

UDG-SMALL5

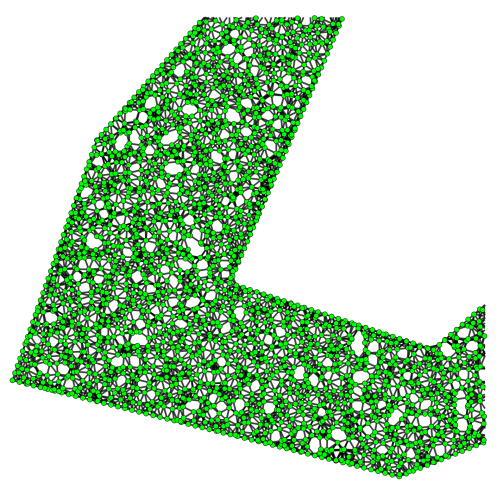

closeup of UDG-LARGE

Fig. 6. Graphs used in our experiments, whose specs are in Table I. All UDG-SMALL graphs have common vertex sets, but different edge densities.

$1,650,2,181$, and 2,809 edges, respectively. The second set of UDG's (UDG-MEDIUM) contained $n=4,563$ vertices in the same polygonal region with max edge length 0.0175 , resulting in $m=19,694$ edges. The third (UDG-LARGE) contained $n=26,542$ vertices in the same polygonal region with max edge length 0.007 , resulting in $m=113,924$ edges. The plane graph PLANAR that we used was a constrained Delaunay triangulation of $n=416$ points scattered randomly within a polygonal region with 12 "holes", resulting in 1,041 edges.

Balanced vertex separators for the nested dissection of the graphs were computed using a simple spectral partitioner, implemented in just 15 lines of MATLAB code. Nested dissection was done up to a cluster with diameter parameter $\delta$ ranging between 1 and 5 . Separators were partitioned into components of parameter size $\gamma$, where $\gamma=1,2,4, \ldots$, up to half the maximal separator size.

For each of the inputs and algorithm parameters, we performed nested dissection, as described in Algorithm 1, and constructed the routing tables CRT and SRT for all graph vertices, as described in Algorithm 3. We then measured the following: (1) average cluster depth in separator tree, (2) average size (number of nodes) of CRT $(v)$, (3) total number of separator components, (4) average size (entries) in $\mathrm{SRT}(v)$, (5) average and standard deviation of stretch of resulting routes. For the small graphs UDG-SMALL and PLANAR, we 


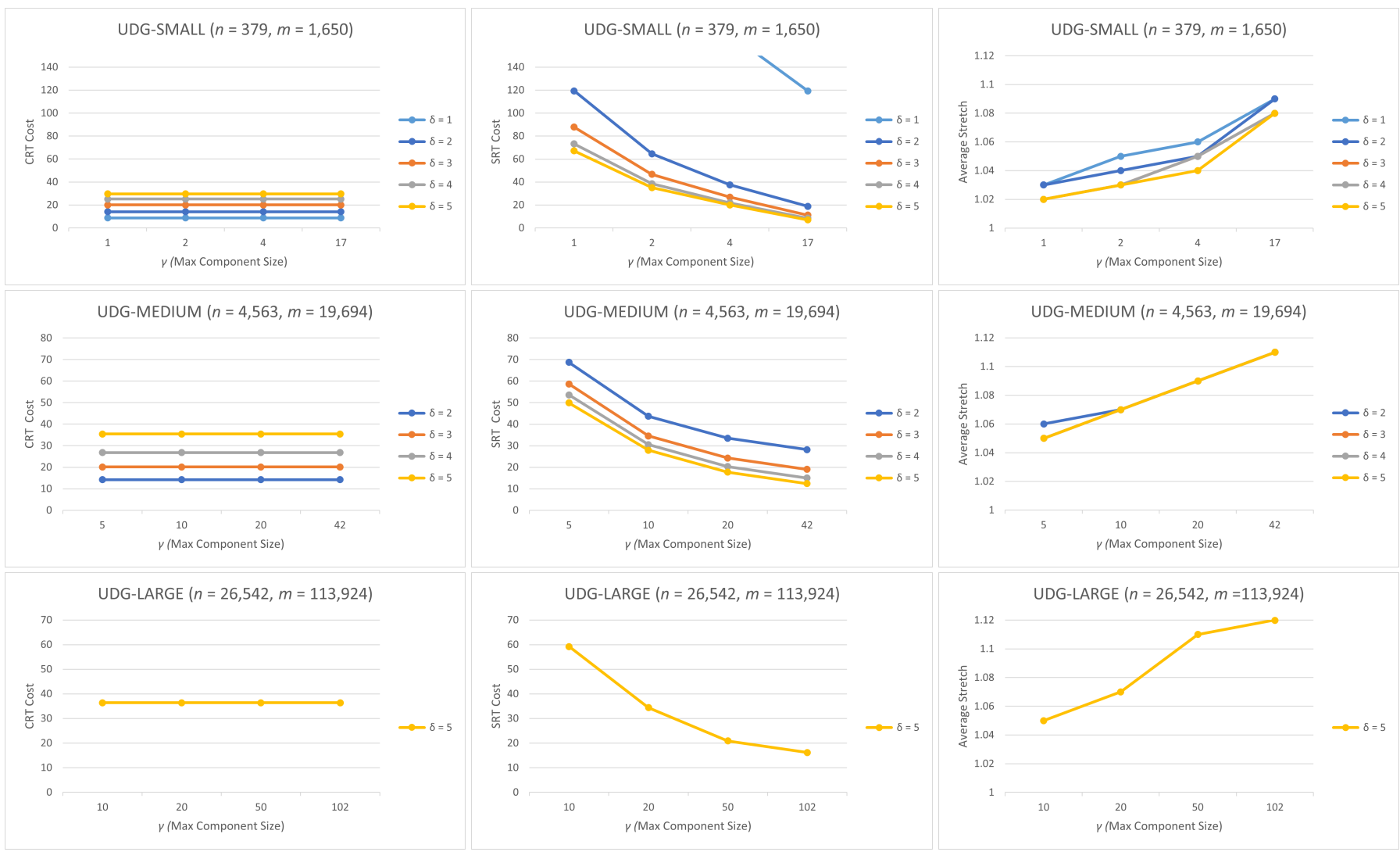

Fig. 7. Average routing table CRT and SRT sizes and stretch for UDG-SMALL, UDG-MEDIUM and UDG-LARGE (at edge density approximately 4.35) vs. max component size $\gamma$ for different values of cluster diameter $\delta$.
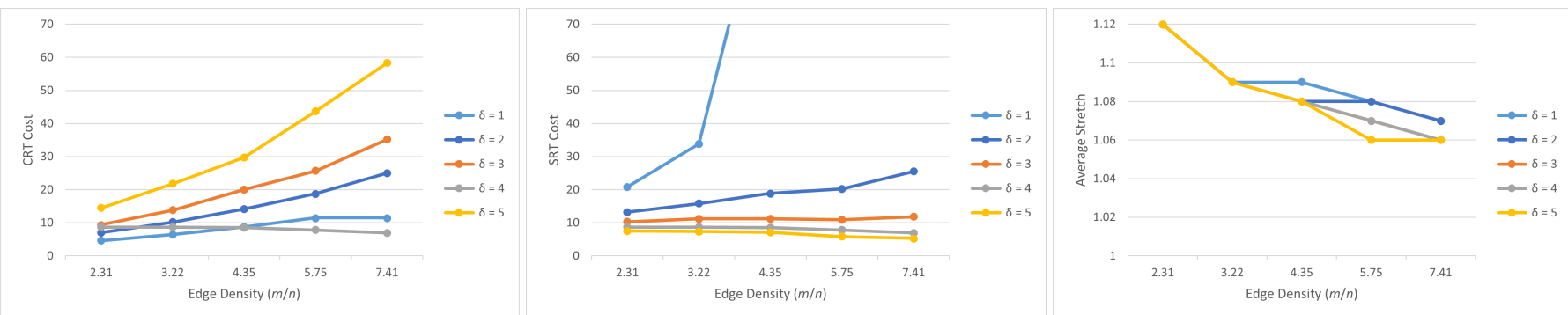

Fig. 8. Average routing table CRT and SRT sizes and stretch for UDG-SMALL vs. edge densities for different values of cluster size diameter $\delta$.

computed (5) and (6) by running our routing algorithm RoUTE (Algorithm 4) exhaustively over all possible $(s, t)$ input pairs of vertices. For the larger graphs (UDG-MEDIUM and UDGLARGE), this was done on a random sample of 1,000 vertex pairs. These graphs and measurements are reported in Table I and Table II.

The cluster diameter parameter $\delta$ determines the termination criterion for the recursive nested dissection. The value 1 means that clusters are cliques. For a triangle mesh, this means that the dissection is done up to the level of individual triangles or edges. Thus, nested dissection of the triangle mesh PLANAR, which contains 614 triangles, will generate a tree of approximately $\log _{2}(614)=9.3$ depth, which is consistent with what we see in Table II. In general, the depth of the separator tree decreases with an increase in this value. This parameter also determines the average size of the CRT part of the routing table per vertex, which will increase with $\delta$. For PLANAR, this will be the average number of neighbors per vertex, namely $2 \times 1,041 / 416=5.0$, also seen in Table II. Partitioning separators into multiple components (as determined by the maximal component size parameter $\gamma$ ) will increase the total number of such components in the system, thus also the average size of the SRT part of the routing table per vertex. However, it will decrease the resulting stretch. So by adjusting the parameters, it is possible to trade off the average size of the routing table: $|\mathrm{CRT}|+|\mathrm{SRT}|$ for stretch. The best stretch (e.g., 1.04 on PLANAR) is obtained when separators are partitioned to components of size one (singletons), however, the cost in routing table size may be prohibitive. A reasonable compromise is obtained for $\delta=4$ and $\gamma=10$, where a stretch of 1.11 is obtained at the price of 14.5 entries in CRT and 13.4 entries in SRT. 

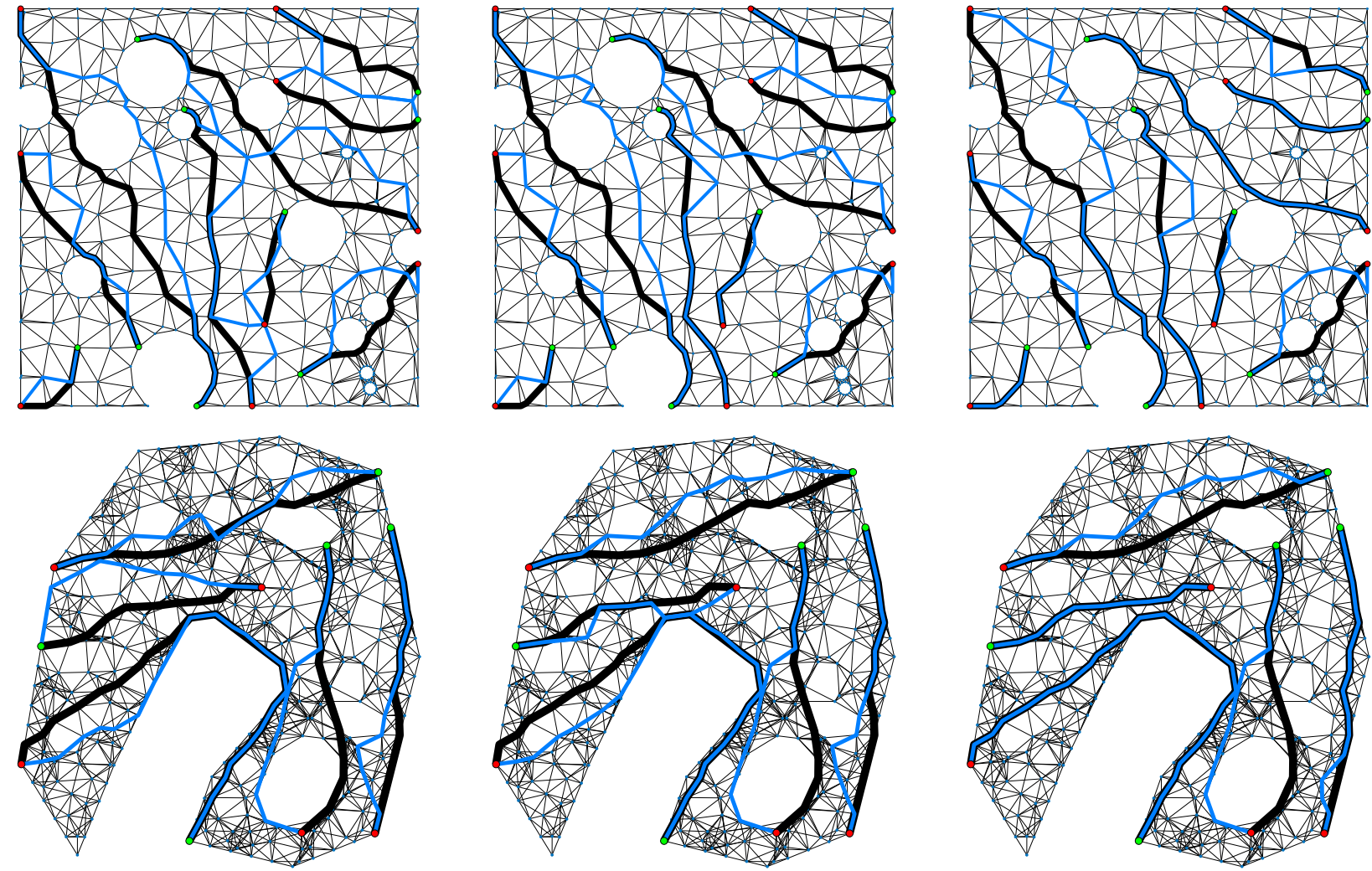

Fig. 9. Sample (blue) paths generated by our compact routing algorithm compared to the true (black) shortest paths for some pairs of (red) $s$ and (green) $t$ vertices. (top, left to right) PLANAR graph with parameters $\delta=1$ and $\gamma=18,4,1$, reducing the stretch. (bottom, left to right) UDG-SMALL3 graph with parameters $\delta=3$ and $\gamma=17,4,1$, reducing the stretch.

TABLE II

EXPERIMENTAL RESULTS ON PLANE GRAPH PLANAR

\begin{tabular}{|c|c|c|c|c|c|c|}
\hline$\delta$ & [Clust. Depth] & [|CRT|] & $\gamma$ & \#Comps & [|SRT|] & [Stretch] \\
\hline \multirow[t]{5}{*}{1} & \multirow[t]{5}{*}{10.0} & \multirow[t]{5}{*}{5.0} & 1 & 1,816 & 133.2 & $1.05 \pm 0.22$ \\
\hline & & & 2 & 1,061 & 74.4 & $1.08 \pm 0.22$ \\
\hline & & & 4 & 844 & 50.7 & $1.10 \pm 0.23$ \\
\hline & & & 10 & 798 & 39.1 & $1.13 \pm 0.24$ \\
\hline & & & 18 & 796 & 37.8 & $1.14 \pm 0.24$ \\
\hline \multirow[t]{5}{*}{2} & \multirow[t]{5}{*}{8.6} & \multirow[t]{5}{*}{8.4} & 1 & 848 & 99.6 & $1.04 \pm 0.08$ \\
\hline & & & 2 & 500 & 55.1 & $1.07 \pm 0.10$ \\
\hline & & & 4 & 332 & 33.4 & $1.09 \pm 0.11$ \\
\hline & & & 10 & 286 & 21.8 & $1.12 \pm 0.13$ \\
\hline & & & 18 & 284 & 20.5 & $1.13 \pm 0.14$ \\
\hline \multirow[t]{5}{*}{3} & \multirow[t]{5}{*}{7.8} & \multirow[t]{5}{*}{11.7} & 1 & 568 & 87.1 & $1.04 \pm 0.08$ \\
\hline & & & 2 & 328 & 47.6 & $1.07 \pm 0.10$ \\
\hline & & & 4 & 203 & 27.9 & $1.09 \pm 0.11$ \\
\hline & & & 10 & 159 & 16.3 & $1.12 \pm 0.13$ \\
\hline & & & 18 & 157 & 15.0 & $1.13 \pm 0.14$ \\
\hline \multirow[t]{5}{*}{4} & \multirow[t]{5}{*}{7.1} & \multirow[t]{5}{*}{14.5} & 1 & 423 & 78.9 & $1.04 \pm 0.08$ \\
\hline & & & 2 & 240 & 42.7 & $1.06 \pm 0.10$ \\
\hline & & & 4 & 144 & 24.8 & $1.09 \pm 0.11$ \\
\hline & & & 10 & 102 & 13.4 & $1.11 \pm 0.13$ \\
\hline & & & 18 & 100 & 12.1 & $1.12 \pm 0.14$ \\
\hline \multirow[t]{5}{*}{5} & \multirow[t]{5}{*}{6.7} & \multirow[t]{5}{*}{19.1} & 1 & 331 & 72.1 & $1.04 \pm 0.08$ \\
\hline & & & 2 & 184 & 38.6 & $1.06 \pm 0.10$ \\
\hline & & & 4 & 110 & 22.4 & $1.08 \pm 0.11$ \\
\hline & & & 10 & 70 & 11.2 & $1.11 \pm 0.13$ \\
\hline & & & 18 & 68 & 9.9 & $1.12 \pm 0.13$ \\
\hline
\end{tabular}

Parameters of the routing table are $\delta$, the maximal cluster diameter in the nested dissection, and $\gamma$, the maximal component size in the separators. The notation $[x]$ indicates the average of quantity $x$. Stretch results are given as average \pm standard deviation.
The charts in Figure 7 report our experimental results on UDG's with increasing vertex size, but a constant edge density of approximately 4.35 edges/vertex (equivalent to an average of 8.7 neighbors per vertex). At this density, taking $\delta=5$ and $\gamma$ to be half of the maximal separator size seems to be a good combination. It is most interesting to see how the resulting average sizes of CRT and SRT scale with the number of vertices. We expect $|\mathrm{CRT}|$ to be somewhat constant, as it depends mostly on $\delta$. Indeed, for $n=4,563$ we have $|\mathrm{CRT}|=35.4$, and for $n=26,542$ we have $|\mathrm{CRT}|=36.4$. On the other hand, we expect $|\mathrm{SRT}|$ to scale logarithmically with $n$. Indeed, for $n=4,563$ we have $|\mathrm{SRT}|=17.7$ and for $n=26,542$ we have $|\mathrm{SRT}|=20.9$, and the difference is quite close to $\log _{2}(26,542 / 4,563)=2.54$. The resulting average stretches are 1.09 and 1.11, respectively.

To compare our results with those of the S4 routing method of Mao et al. [4], we state the conclusion from their experiments that to achieve stretch between 1.05 and 1.10 with S4 requires the storage requirements to scale like $O(\sqrt{n})$.

It is interesting to study the tradeoffs when the edge density of the graph is increased. There it would be beneficial to use clusters of larger diameter $\delta$. Note that as the density increases, these clusters tend to be larger (in vertex count), thus the size of CRT will increase.

Figure 9 compares some of the paths generated by our compact routing algorithm with the true shortest paths between pairs of vertices. Figures 10 and 11 compare the complete compact routing trees on some select vertices to the shortest- 

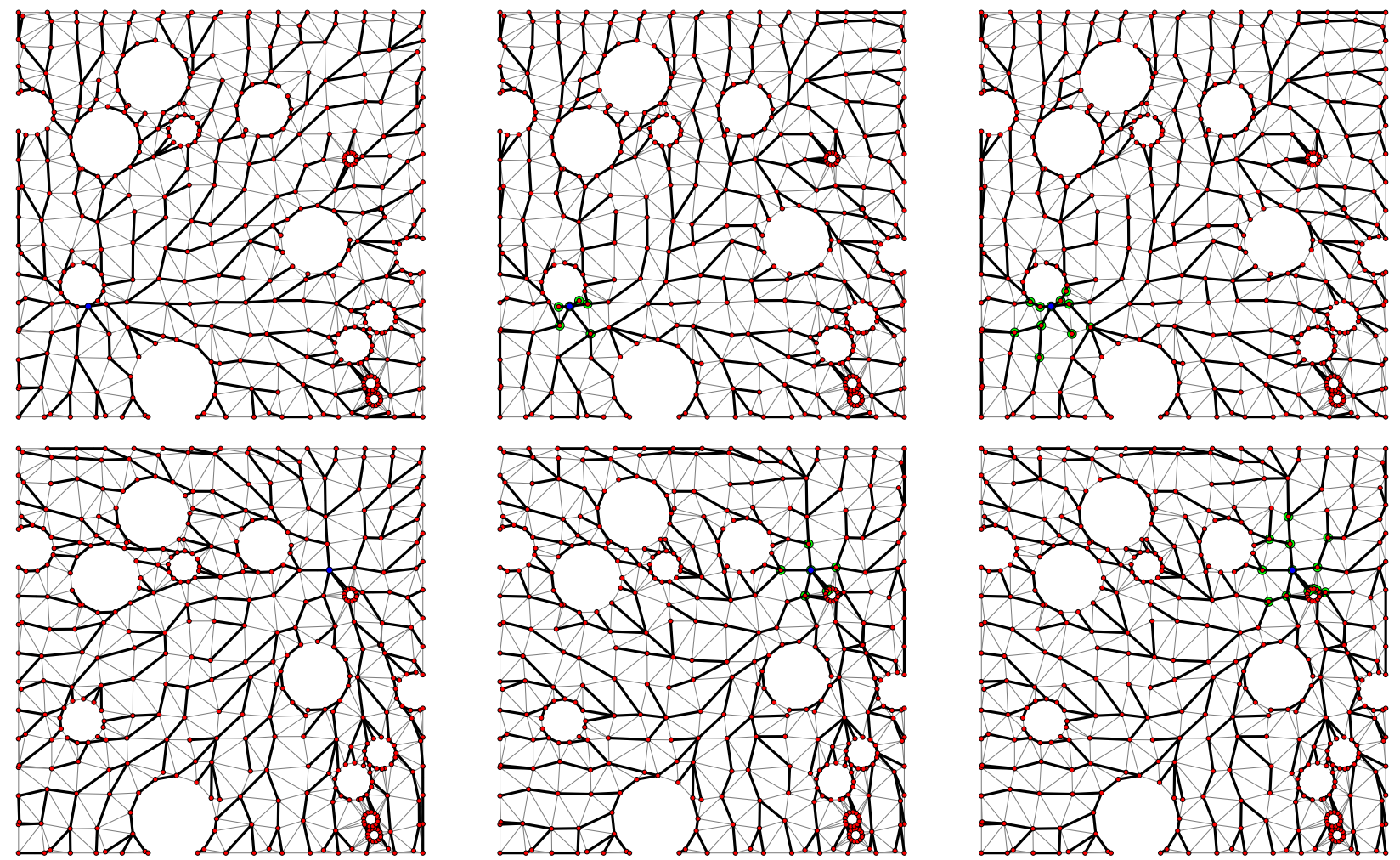

Fig. 10. Comparison of routing trees for two arbitrary (blue) target vertices of the PLANAR graph: shortest-path routing trees (left) versus routing trees generated by our compact routing algorithm for same target vertices with $\delta=1$ (middle) and $\delta=2$ (right), both with $\gamma=18$. Green halos mark the vertices in a common cluster with $t$.
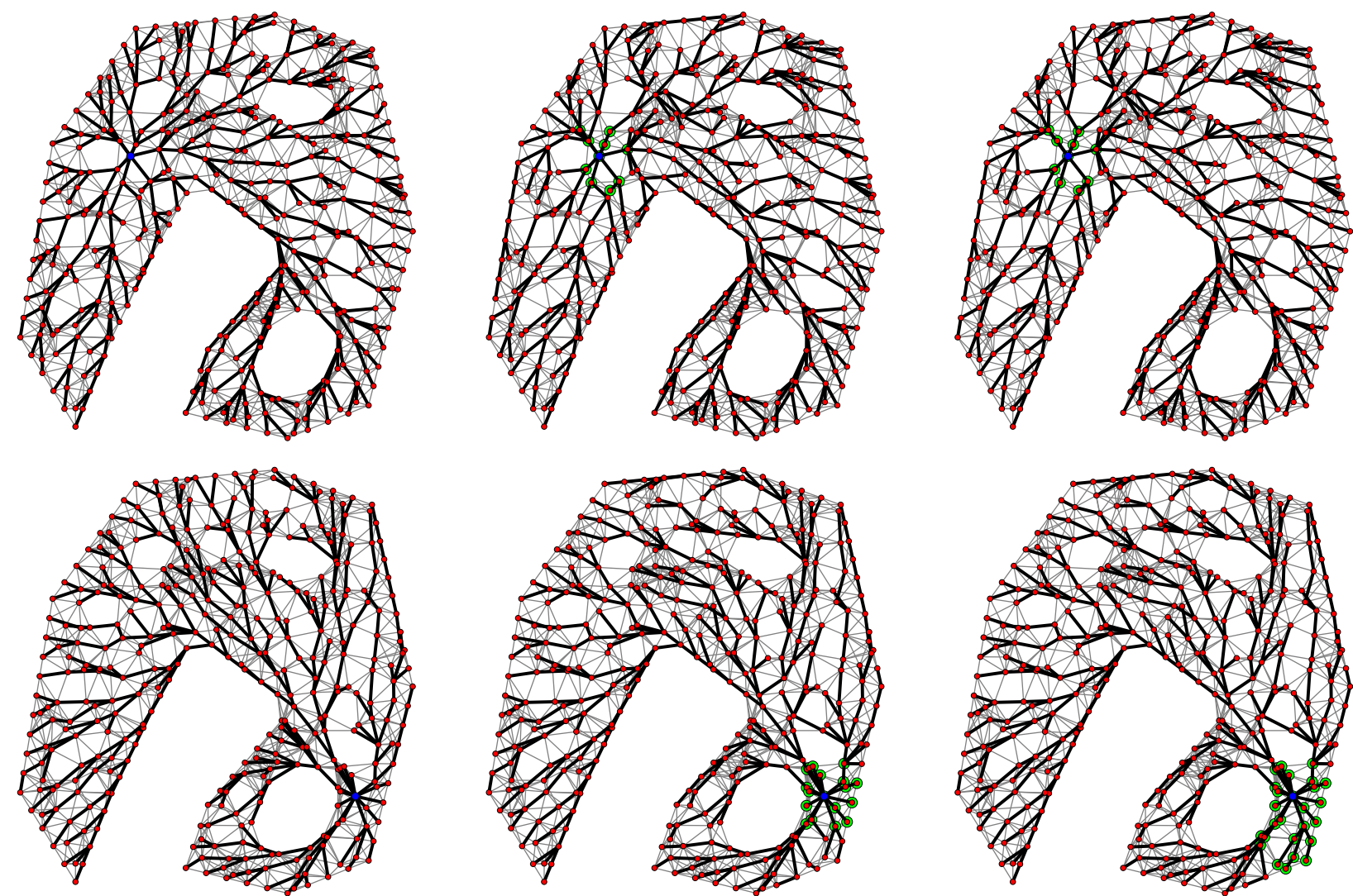

Fig. 11. Comparison of routing trees for two arbitrary target vertices of the UDG-SMALL3 graph: shortest-path routing trees (left) versus routing trees generated by our compact routing algorithm for same target vertices with $\delta=2$ (middle) and $\delta=3$ (right), both with $\gamma=17$. Green halos mark the vertices in a common cluster with $t$. 
path routing trees for those same targets.

\section{Discussion}

We have described a simple, yet effective, algorithm to construct compact routing tables for graphs which have compact balanced vertex separators. Prime examples of these are planar graphs and UDG's with moderate density, the so-called sweet spot. Our method is not suitable for very sparse UDG's, since then, even if $G$ is connected, the separation routine may result in two components which are not necessarily themselves connected. This may prevent the router finding any path between $s$ and $t$, even if such a path exists in $G$. Our method is also not suitable for UDG's with high density, since these do not admit sparse separators, and become increasingly difficult to separate as the nested bisection digs down to subgraphs which are almost cliques. This may be alleviated somewhat by increasing $\delta$, which terminates the recursion at larger clusters, before they become too dense, but there will still be a price to pay in the size of CRT, which routes within these clusters. Some estimates of the size of clusters of diameter $\delta$ in UDG's (sometimes also called $\delta$-clubs) may be found in the random graph literature [24].

The efficiency of the resulting routing tables in terms of the state-stretch tradeoff depends on the quality of these separators. In our implementation, we used simple methods which generate reasonable separators, but this may be further improved by using a more sophisticated algorithm to improve the quality of the separators. For example, in the case of planar graphs, separators are typically paths of vertices. If they can be further guaranteed to be shortest paths between their endpoints, then it is easy to see that better stretch can be achieved, since, in this case, the shortest path between two vertices on one side of the separator is guaranteed to be contained completely in that part of the graph. In particular, the stretch can theoretically reach the perfect 1 if each separator is partitioned into singleton components $(\gamma=1)$. This, however, will produce routing tables of size $O(\sqrt{n})$.

We note that in our experiments, graph distance and path length were measured on graphs whose edges were weighted with their Euclidean edge lengths. However, for uniform edges length (i.e., a combinatorial graph), we do not expect anything significant to change in our results. Finally, we should point out that our method is not suitable for dynamic graphs, where the connectivity or the edge weights $w$ vary over time, since any such change would require the routing tables to be recomputed.

\section{REFERENCES}

[1] P. Carmi, M. K. Chiu, M. J. Katz, M. Korman, Y. Okamoto, A. van Renssen, M. Roeloffzen, T. Shiitada, and S. Smorodinsky, "Balanced line separators of unit disk graphs," Comput. Geom., vol. 86, pp. 101 575:114, Jan. 2020.

[2] R. J. Lipton and R. E. Tarjan, "A separator theorem for planar graphs," SIAM J. Appl. Math., vol. 36, no. 2, pp. 177-189, 1979.

[3] M. Thorup and U. Zwick, "Compact routing schemes," in Proceedings of the Thirteenth Annual ACM Symposium on Parallel Algorithms and Architectures, 2001, pp. 1-10.

[4] Y. Mao, F. Wang, L. Qiu, S. Lam, and J. Smith, "S4: Small state and small stretch compact routing protocol for large static wireless networks," IEEE/ACM Trans. Netw., vol. 18, no. 3, pp. 761-774, Jun. 2010.
[5] M. Dom, "Compact routing," in Algorithms for Sensor and Ad Hoc Networks, ser. Lecture Notes in Computer Science, D. Wagner and R. Wattenhofer, Eds. Berlin, Heidelberg: Springer, 2007, vol. 4621, pp. 187-202.

[6] G. N. Frederickson and R. Janardan, "Efficient message routing in planar networks," SIAM J. Comput., vol. 18, no. 4, pp. 843-857, Aug. 1989.

[7] M. Thorup, "Compact oracles for reachability and approximate distances in planar digraphs," J. ACM, vol. 51, no. 6, pp. 993-1024, Nov. 2004.

[8] I. Abraham, C. Gavoille, and D. Malkhi, "Compact routing for graphs excluding a fixed minor," in Distributed Computing, ser. Lecture Notes in Computer Science, P. Fraigniaud, Ed. Berlin, Heidelberg: Springer, 2005, vol. 3724, pp. 442-456.

[9] H.-I. Lu, "Improved compact routing tables for planar networks via orderly spanning trees," SIAM J. Discrete Math., vol. 23, no. 4, pp. 2079-2092, 2010.

[10] A. Gupta, A. Kumar, and R. Rastogi, "Traveling with a pez dispenser (or, routing issues in MPLS)," SIAM J. Comput., vol. 34, no. 2, pp. 453-474, 2005.

[11] C. Yan, Y. Xiang, and F. F. Dragan, "Compact and low delay routing labeling scheme for Unit Disk Graphs," Comput. Geom., vol. 45, no. 7, pp. 305-325, Aug. 2012.

[12] H. Kaplan, W. Mulzer, L. Roditty, and P. Seiferth, "Routing in unit disk graphs," Algorithmica, vol. 80, no. 3, pp. 830-848, Mar. 2018.

[13] W. Mulzer and M. Willert, "Routing in unit disk graphs without dynamic headers," arXiv:2002.10841v1, 2020

[14] C. Wulff-Nilsen, "Approximate distance oracles for planar graphs with improved query time-space tradeoff," in Proceedings of the TwentySeventh Annual ACM-SIAM Symposium on Discrete Algorithms, 2016, p. $351-362$.

[15] P. Gawrychowski, S. Mozes, O. Weimann, and C. Wulff-Nilsen, "Better tradeoffs for exact distance oracles in planar graphs," in Proceedings of the Twenty-Ninth Annual ACM-SIAM Symposium on Discrete Algorithms, 2018, pp. 515-529.

[16] P. Bose and P. Morin, "Online routing in triangulations," SIAM $J$. Comput., vol. 33, pp. 937-951, 2004.

[17] C. H. Papadimitriou and D. Ratajczak, "On a conjecture related to geometric routing," Theor. Comput. Sci., vol. 344, p. 3-14, 2005.

[18] M. Ben Chen, S. J. Gortler, C. Gotsman, and C. Wormser, "Distributed computation of virtual coordinates for greedy routing in sensor networks," Discrete Appl. Math., vol. 159, pp. 544-560, 2011.

[19] N. Bonichon, P. Bose, J.-L. De Carufel, V. Despré, D. Hill, and M. Smid, "Improved routing on the Delaunay triangulation," in 26th Annual European Symposium on Algorithms (ESA 2018), ser. Leibniz International Proceedings in Informatics (LIPIcs), Y. Azar, H. Bast, and G. Herman, Eds., vol. 112, 2018, pp. 22:1-22:13

[20] A. George, "Nested dissection of a regular finite element mesh," SIAM J. Numer. Anal., vol. 10, no. 2, pp. 345-363, 1973.

[21] D. A. Spielman and S.-H. Teng, "Spectral partitioning works: Planar graphs and finite element meshes," Linear Alg. Appl., vol. 421, no. 2-3, pp. 284-305, Mar. 2007.

[22] A. Buluç, H. Meyerhenke, I. Safro, P. Sanders, and C. Schulz, "Recent advances in graph partitioning," in Algorithm Engineering, ser. Lecture Notes in Computer Science, L. Kliemann and P. Sanders, Eds. Cham: Springer, 2016, vol. 9220, ch. 3, pp. 117-158.

[23] "METIS: Serial graph partitioning and fillreducing matrix ordering." [Online]. Available: http://glaros.dtc.umn.edu/gkhome/metis/metis/download

[24] R. B. Ellis, X. Jia, and C. Yan, "On random points in the unit disk," Random Struct. Algorithms, vol. 29, no. 1, pp. 14-25, Aug. 2006. 